\title{
Risk Assessment for ITER TF Coil Manufacturing*)
}

\author{
Kenichi UENO, Kunihiro MATSUI, Toru NISHINO, Takaaki ISONO and Kiyoshi OKUNO \\ Japan Atomic Energy Agency, 801-1 Mukoyama, Naka 311-0193, Japan
}

(Received 7 December 2012 / Accepted 7 March 2013)

\begin{abstract}
The Japan Domestic Agency (JADA) for ITER will procure toroidal field (TF) coil structures and winding packs and assemble them into a final TF coil configuration. Because the manufacturing schedule of the TF coils is a critical path toward the first plasma of ITER, coil manufacturing must be successful and proceed on schedule. Therefore, risk assessment and management for its manufacturing are essential. JADA performed a risk assessment on the basis of past manufacturing experiences and a risk mitigation policy for ITER-TF coil manufacturing. The results show that risks can be mitigated to a level that we can assure sufficient quality of the TF coil by sound design, manufacturing, and quality management processes that are developed through $R \& D$ activities and the use of prototypes.
\end{abstract}

(c) 2013 The Japan Society of Plasma Science and Nuclear Fusion Research

Keywords: ITER, toroidal field coil, superconducting magnet, risk assessment, risk mitigation

DOI: $10.1585 /$ pfr.8.2405062

\section{Introduction}

The ITER superconducting (SC) magnet system contains 18 toroidal field (TF) coils, a central solenoid (CS) consisting of six modules, and six poloidal field (PF) coils [1]. The coils produce a toroidal magnetic field to confine plasma in a tokamak. The Japan Atomic Energy Agency acts as the Japan Domestic Agency (JADA) in the ITER project; it is responsible for 19 (18 and one spare) TF coil structures, nine winding packs (WP), and the assembly of the WP and the coil structure for nine of the 19 coils [2]. The ITER TF coil design is shown in Fig. 1. Because the manufacturing schedule for the TF coils is the critical path toward the first plasma, manufacturing must proceed successfully and on schedule. If there is any trouble in the TF coil after assembly, repair work or replacement would be expensive and result in a half-year delay in the schedule. Any problems related to the manufacturing of the TF coil must be avoided; such a risk must be mitigated by sound design, manufacturing methods, and quality assurance / quality control (QA/QC) processes, developed through $R \& D$ activities and prototypes. These steps are to be confirmed before the manufacture of the actual product. In addition, risk assessment and management for the TF-coil-manufacturing process are essential for the ITER project.

\section{Risk Analysis}

The first step in risk analysis is to identify risk sources. Next, risk mitigation plans and actions are considered. Four main risk categories were identified in TF-coil manufacturing: helium leaks, insulation, joints, and instrumen-

author'se-mail: ueno.kenichi@jaea.go.jp

*) This article is based on the presentation at the 22nd International Toki Conference (ITC22).

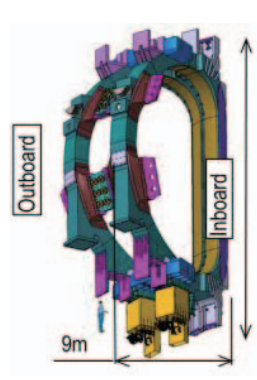

A pair of TF coils

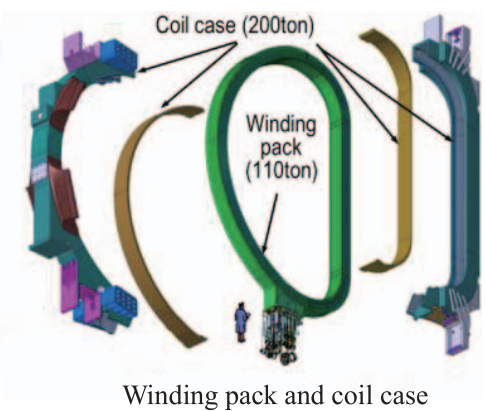

Fig. 1 ITER TF coil design [2].

tation.

For quantitative risk assessment, JADA performed research on the manufacture of SC magnets in the Korea Superconducting Tokamak Advanced Research (KSTAR) and the Japanese cryogenic industry experiences. KSTAR was built at the National Fusion Research Institute in Daejon, South Korea; it contains $30 \mathrm{SC}$ coils [3]. In KSTAR, manufacturing procedures and QA/QC processes were developed through $R \& D$ activities, and mock-ups were fabricated to establish manufacturing and QA/QC processes. Actual coils were manufactured by these established procedures and processes. No cold tests were performed for the actual coils. The KSTAR magnets exhibited no helium leaks, joint failure, or insulation failure.

JADA evaluated risk and its mitigation for each of the four main risks as follows: 1) identification of potential risk source; 2) risk mitigation; and 3) evaluation of risk likelihood. Risk mitigation further consisted of the following steps, taken before actual production commenced: a) risk mitigation by sound design (the use of techniques proven by ITER model coils); b) risk mitigation by use of prototypes (in pre-fabrication, a prototype would be de- 
veloped to validate and demonstrate the techniques); and c) risk mitigation during production by the application of stringent QA/QC procedures (these QA/QC procedures are demonstrated during the production of the mock-up coil); the actual TF coils will be checked by well-established $\mathrm{QA} / \mathrm{QC}$ processes to verify the final quality of the coils.

\section{Assessment Details}

\subsection{Helium leaks}

\subsubsection{Design and potential risks}

A WP has five regular double pancakes (DPs) that use a 760-m conductor and two side DPs using 430-m conductors. Since each conductor jacket is welded in a $13-\mathrm{m}$ jacketing section, there are around 350 welds of the conductor jacket in a WP. On the other hand, supercritical helium is supplied from a helium inlet, which is located between pancakes, and exits from two helium outlets at both ends of the conductor. Therefore, the helium circuit of the TF coil consists of seven conductors, seven helium inlets, and 14 helium outlets. The helium inlets and outlets are located at the bottom of the TF coil, and these areas are accessible after tokamak assembly.

\subsubsection{Risk mitigation}

The risk will be mitigated as follows:

a) Risk mitigation in design and manufacturing-Every jacket-welding part will be nondestructively tested by multiple methods (helium leak test, pressure-proof test, dye penetration test, and radiographic testing). Only accepted welding parts will be used for the conductor jacket. All helium inlet and outlet welded parts will be tested (helium leak test and pressure-proof test). Only those helium inlet and outlet welded parts that pass the tests will be used.

Helium inlets, outlets, and the jacket-welding part will be exposed to heat treatment performed at $650^{\circ} \mathrm{C}$ for $200 \mathrm{~h}$, which is adequate for preventing cold leaks. Argon gas will be filled in the conductor and vacuum in the oven during heat treatment. Because the heat treatment introduces higher thermal stresses (more than a $600^{\circ} \mathrm{C}$ temperature difference) than cold stresses (about a $300^{\circ} \mathrm{C}$ temperature difference in real operational conditions), and activated molecules will attack welding parts at high temperature $\left(650^{\circ} \mathrm{C}\right)$, all possible welding parts leaks can be detected by monitoring pressure and inspecting the gas leaks in the oven during heat treatment and a subsequent leak test performed at room temperature.

b) Risk mitigation using prototypes-CS Model coil inserts were successfully manufactured and tested at ITEREDA and exhibited no leaks. The manufacturing method and procedure were verified.

Full-scale mock-up ITER TF coils for helium inlets and outlets (terminal area) will be developed by suppliers to demonstrate the sound design and manufacturing process (including welding methods and inspection methods) before actual manufacturing. These mock-ups must be passed through repeated cyclic fatigue tests; these tests simulated an electric magnetic force that is 10 times stronger than thermal stress. Thus, a repeated thermal cycle stress test is covered by a fatigue test employing full scale mock-up.

c) Risk mitigation by QA/QC — Parts will be welded in accordance with the qualified manufacturing processes and QA/QC procedures. Welds will be repeatedly tested at different manufacturing phases. The first DP will be tested after a thermal cycle up to $77 \mathrm{~K}$ to confirm manufacturing and QA/QC procedures and to improve them if necessary.

\subsubsection{Examples}

JADA investigated cold leaks in the following fusion SC magnets: the TF and CS model coils in ITER-EDA [4-6], Large Helical Device (LHD) at the National Institute for Fusion Science [7], Demo Poloidal Coils at JAEA (DPC) [8-10], and KSTAR. The numbers of joints and terminals, including helium inlets or outlets, were as follows: TF and CS model coils, 86; LHD, 54; DPC, 17; and KSTAR, 64 terminals, 327 joints including busbars.

Only the TF model coil exhibited a cold leak; recognition of this is important for the improvement of the QC process [4]. The assembly of the CS model coil also had a cold leak at a corner weld between the cooling pipe and support structure (not at the welding joint of the pipe) [6]. This was an example of improper implementation of QA/QC. The welding was of poor quality; a repair weld was performed at the factory without a proper review of procedures, and the customer was not informed of it until the cold leak occurred.

JADA studied the experiences of the Japanese cryogenic industry with regard to helium leaks. Japanese cryogenic suppliers reported that more than 30,000 welding joint parts were fabricated and tested. The risk likelihood was estimated as number of failures divided by total number of products in this study. Based on this study, the maximum risk likelihood is estimated to be $1.5 \%$ per system in the case of no cold tests; this could be reduced to around $0.03 \%$ per system if cold tests were done.

In the previous manufactured fusion SC magnets, conductor jackets requiring welding parts in a much simpler shape than terminal or joint parts, exhibited no cold leaks upon testing. Conductor-jacket-welding parts have far fewer leaks than terminal or joint parts. A good example is KSTAR, which has a total of 391 terminals and joints but no cold leaks at $4.2 \mathrm{~K}$. That the definition of maximum risk likelihood is the hypothetical failure of the 392nd part. The risk likelihood for welding part or joint failure was estimated as the inverse of 392 , around $0.25 \%$ per system with inspection.

\subsubsection{Risk evaluation}

The failure risk likelihood was evaluated for three classes: failure that happens at accessible areas, inaccessible areas, and inaccessible areas with replacement by a 
new coil. If a leak happens at an accessible area, repair work would be done in the cryostat, and it would take under a year; the risk likelihood is less than $0.5 \%$ per system, which eliminates the KSTAR value $(0.3 \%)$. If a leak happens at an inaccessible area, the magnets would have to be moved for repair work in the cryostat, and it would take about one and a half years; this event's risk likelihood is quite small, because terminals and joints, which are at a higher risk for helium leaks, are located in accessible areas of the tokamak. The risk likelihood is considered to be one-tenth that of the first value. However, this value is not based on data from experience; rather, it is the result of multiplication of 0.05 by a safety factor of $2.5(<0.125 \%)$. The risk that a magnet would need to be removed for replacement by a new coil is extremely small; therefore, the likelihood is one-tenth that of second one $(<0.0125 \%)$.

\subsection{Joints}

\subsubsection{Design and potential risks}

Each TF coil has seven conductors, two terminal joints, and six internal joints. Joints at the terminal area are shown in Fig. 2. The two ends of each conductor will be shaped in a joint configuration before heat treatment to generate an $\mathrm{Nb}_{3} \mathrm{Sn}$ superconductor. Subsequently, the internal joints will be assembled.

Joint resistance could increase for the following reasons: damaged $\mathrm{Nb}_{3} \mathrm{Sn}$ strands during manufacturing; bad bonding between a cable and a copper and/or between copper; or insufficient support of the joints for resisting operational loads.

\subsubsection{Risk mitigation}

a) Risk mitigation by design-Joint design and manufacturing methods have been demonstrated and verified, and good bonding and sufficiently low joint resistance were confirmed by the SULTAN test.

b) Risk mitigation using prototypes and by QA/QCManufacturing processes have been established and demonstrated at the industry level for the CS and TF model coils. Mock-up joints will be fabricated and tested at $4.2 \mathrm{~K}$ to demonstrate these processes. A manufacturing process including $\mathrm{QA} / \mathrm{QC}$ procedures was established and confirmed by prototype production before the actual production.

\subsubsection{Risk likelihood evaluation}

JADA researched joint performance in the fusion SC magnets described in Section 3.1.3 and found no joint resistance failure. The risk likelihood of joint failure at accessible areas was found to be significantly low $(<0.1 \%)$. The risk at an inaccessible area and need for a magnet to be removed for replacement by a new coil is extremely small (there is no joint-inaccessible area); therefore, the likelihood is one-tenth the above value $(<0.01 \%)$.

\subsection{Insulation}

\subsubsection{Design and potential risks}

TF coils have three insulation systems, as shown in Fig. 3: conductor insulation between a conductor and a radial plate (RP), DP insulation between two DPs, and ground between a conductor and an RP (defects in conductor insulation). All are potential risks; they may cause earth faults and/or short-circuit faults. Defects in ground insulation covering conductors or pipes outside RPs also are at potential risk for insulation faults.

\subsubsection{Risk mitigation}

a) Risk mitigation by design-The insulation system consists of multiple layers of polyimide film glass impregnated with epoxy resin. The use of polyimide film guarantees good results. In addition, the conductor, DP, and ground insulation system are independent and physically separated. Therefore, it is impossible for a single insulation fault to affect them all. In particular, the conductor insulation is mechanically protected by RPs; therefore, no direct path exists for electromagnetic loads from a conductor to the DP or ground insulation. Each insulation system will be inspected when it is applied, and repair is possible at this stage.

b) Risk mitigation using prototypes-The design concept of the insulation system was demonstrated in the TF and CS model coils, including several cool-down and energization cycles. As part of the insulation system demonstration, JAEA fabricated a one-third-scale mock-up (Fig. 4). The mock-up withstood the required high voltage; no voids or damage in the insulation appeared in a cross section of the mock-up (Fig. 5). JADA will develop a full-scale DP and mock-ups of critical areas to verify insulation procedures. The full-scale DP will be cold-tested at $77 \mathrm{~K}$.

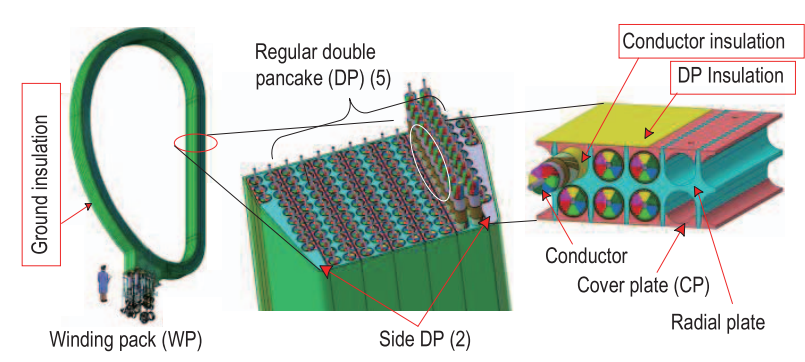

Fig. 2 Joints at terminal area.

Fig. 3 TF coil insulation system. 


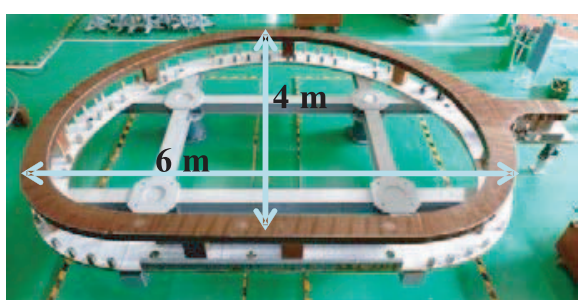

Fig. 4 Outer view of one-third-scale mock-up.

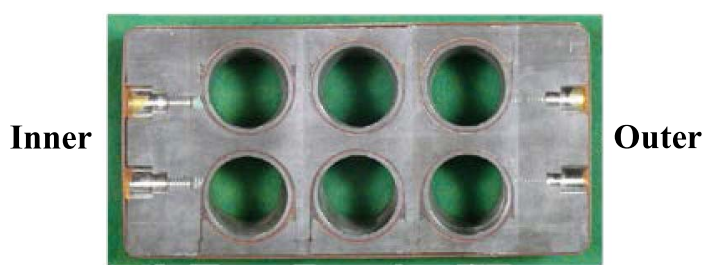

Fig. 5 Cross-sectional surface of mock-up.

c) Risk mitigation by $\mathrm{QA} / \mathrm{QC}-\mathrm{QA} / \mathrm{QC}$ activities will include high-voltage tests to confirm insulation performance after each manufacturing process. The first, second, and third inspections will be performed after the DP acceptance test. In each inspection, insulation faults can be detected by a high-voltage test and repaired as described in a) above. The first DP will be tested after a thermal cycle up to $77 \mathrm{~K}$ to confirm manufacturing and QA/QC procedures and to improve them if necessary.

\subsubsection{Risk evaluation}

JAEA investigated insulation failure in the fusion SC magnets described in Section 3.1.4. Only the TF model coil exhibited insulation failure; experience with this model coil is important for improving QC processes.

A Japanese industrial SC magnet supplier reported that it has supplied more than 600 magnets using NbTi and $\mathrm{Nb}_{3} \mathrm{Sn}$ SC wires, with a risk likelihood of $0.15 \%$ in insulation failure with inspection.

Although the TF model coil exhibited a localized insulation failure, the TF model coil at KSTAR offers a successful example. In addition, industrial experience indicates very low risk likelihood. Consequently, the risk likelihood of insulation defects in accessible areas is $0.15 \%$, known from industrial experience and from removal of a magnet for external repair or replacement with a spare is basically the same $(0.15 \%)$. However, the catastrophic risk of removing a magnet for replacement with a new coil is considered to be extremely small, one-tenth the above value $(<0.015 \%)$.

\subsection{Instrumentation}

\subsubsection{Design and potential risks}

Each TF coil includes the following instrumentation: quench detection (QD) tapes wound on conductors; voltage taps attached near joints and terminals; and flow me- ters, pressure gauges, check valves, flow-balancing valves, and thermometers on pipes.

Potential risks to the instrumentation are insulation faults in QD tapes and voltage taps, helium leaks at flow meters, pressure gauges, check valves and flow-balancing valves, sensor malfunctions, and wire breaks anywhere in the instrumentation. The malfunction of thermometers and their wire breaks must also be considered.

\subsubsection{Risk mitigation}

Risk mitigation methods for insulation failure and helium leaks are described above. Flow meters, pressure gauges, check valves, flow-balancing valves, and thermometers will be attached to ground-voltage pipes and installed after the coil casing manufacturing process. They can be separately cold-tested at $4 \mathrm{~K}$ before installation to mitigate the risk. Thermometer and their wires can be installed with sufficient redundancy to mitigate the risk of breakage.

\subsubsection{Risk evaluation}

Flow meters, pressure gauges, check valves, flowbalancing valves, and thermometers are located in accessible areas for repair even in the tokamak; magnet removal for external repair is very rare. The risk likelihood of instrumentation problems was evaluated as $0.5 \%$ for accessible areas and $0.02 \%$ for inaccessible areas.

\section{Assessment Result}

JADA performed risk assessment for four main TFcoil manufacturing risks: helium leaks, insulation, joints and instrumentation. The results show that the risks in TF coil manufacture can be mitigated by sound design, manufacturing methods, and QA/QC processes that are developed through $R \& D$ activities and prototypes.

On the basis of this study, JADA will implement the following activities for risk mitigation: 1) manufacturing of prototypes for critical areas for helium leak tightness and insulation integrity, 2) a 77-K test for a dummy DP and the first-of-series DPs (DPs 1-3), and 3) application of well-established, stringent QA/QC processes based on past experience and the prototype activities. The implementation of these assures the quality of the TF coils required for ITER.

\section{Acknowledgment}

The authors thank Dr. E. Tada and Dr. M. Sugimoto of JAEA for their continuous support of this study. The authors also express their deep appreciation to Dr. K. Jung, Dr. J.S. Bak, Dr. K. Kim, Dr. P. Kaprai, Dr. H.Y. Yang, Dr. Y.M. Park, and Dr. J. Ko of the Korean National Fusion Research Institute for sharing their valuable KSTAR experience and for fruitful, collaborative discussions regarding this study. The authors would like to thank Enago (www.enago.jp) for the English language review. 
[1] N. Mitchell et al., IEEE Transactions 18, 2 (2008).

[2] N. Koizumi et al., Fusion Eng. Des. 84, 210 (2009).

[3] K.R. Park et al., IEEE Transactions 19, 3 (2009).

[4] N. Mitchell, Fusion Eng. Des. 66-68, 971 (2003).

[5] A. Ulbricht et al., Fusion Eng. Des. 73, 189 (2005).

[6] H. Tsuji et al., Fusion Eng. Des. 55, 153 (2001).
[7] S. Imagawa et al., Fusion Eng. Des. 41, 253 (1998).

[8] H. Tsuji et al., Proc. MT-11 Elsevier Science Publisher, Essex, UK (1990).

[9] K. Okuno et al., Proc. MT-11 Elsevier Science Publisher, Essex, UK (1990).

[10] T. Ando et al., IEEE Transactions 27, 2 (1991). 